OPEN ACCESS

Edited by:

David A. Gewirtz,

Virginia Commonwealth University,

United States

Reviewed by:

Hisashi Harada,

Virginia Commonwealth University,

United States

Jingwen Xu,

Guangdong Pharmaceutical

University, China

*Correspondence:

Yanfeng Chen

chenyf@sysucc.org.cn

Xiaoping Lin

linxp@sysucc.org.cn

these authors have contributed equally to this work

Specialty section: This article was submitted to Pharmacology of Anti-Cancer Drugs, a section of the journal Frontiers in Pharmacology

Received: 14 November 2020 Accepted: 02 March 2021

Published: 13 April 2021

Citation:

Hao W, Wu L, Cao L, Yu J, Ning L, Wang J, Lin $X$ and Chen Y (2021)

Radioresistant Nasopharyngeal

Carcinoma Cells Exhibited Decreased

Cisplatin Sensitivity by Inducing

SLC1A6 Expression.

Front. Pharmacol. 12:629264.

doi: 10.3389/fphar.2021.629264

\section{Radioresistant Nasopharyngeal Carcinoma Cells Exhibited Decreased Cisplatin Sensitivity by Inducing SLC1A6 Expression}

\author{
Wenwen $\mathrm{Hao}^{1,2 \dagger}$, Lisha $\mathrm{Wu}^{3 \dagger}$, Linhui Cao ${ }^{4}$, Jinxiu $\mathrm{Yu}^{5}$, $\mathrm{Li} \mathrm{Ning}^{2}$, Jingshu Wang ${ }^{3}$, \\ Xiaoping Lin $^{6 * t}$ and Yanfeng Chen ${ }^{2 *}$
}

${ }^{1}$ Department of Nasopharyngeal Carcinoma, State Key Laboratory of Oncology in South China, Collaborative Innovation Center for Cancer Medicine, Guangdong Key Laboratory of Nasopharyngeal Carcinoma Diagnosis and Therapy, Sun Yat-sen University Cancer Center, Guangzhou, China, ${ }^{2}$ Department of Head and Neck Surgery, State Key Laboratory of Oncology in South China, Collaborative Innovation Center for Cancer Medicine, Sun Yat-sen University Cancer Center, Guangzhou, China, ${ }^{3}$ Department of Oncology, Sun Yat-sen Memorial Hospital, Sun Yat-sen University, Guangzhou, China, ${ }^{4}$ Department of Traditional Chinese Medicine, Sun Yat-sen Memorial Hospital, Sun Yat-sen University, Guangzhou, China, ${ }^{5}$ Department of Radiotherapy, The Second Affiliated Hospital of Guangzhou Medical University, Guangzhou, China, ${ }^{6}$ Department of Nuclear Medicine, State Key Laboratory of Oncology in South China, Collaborative Innovation Center for Cancer Medicine, Sun Yat-sen University Cancer Center, Guangzhou, China

Cisplatin-based regimens are commonly used for the treatment of nasopharyngeal carcinoma (NPC) in patients who receive concurrent chemoradiotherapy. The sensitivity of NPC cells to cisplatin is closely associated with the efficacy of radiation therapy. In this study, we established two radioresistant NPC cell lines, HONE1-IR and CNE2-IR, and found that both cell lines showed reduced sensitivity to cisplatin. RNAsequence analysis showed that SLC1A6 was upregulated in both HONE1-IR and CNE2-IR cell lines. Downregulation of SLC1A6 enhanced cisplatin sensitivity in these two radioresistant NPC cell lines. It was also found that the expression of SLC1A6 was induced during radiation treatment and correlated with poor prognosis of NPC patients. Notably, we observed that upregulation of SLC1A6 led to elevating level of glutamate and the expression of drug-resistant genes, resulted in reduced cisplatin sensitivity. Our findings provide a rationale for developing a novel therapeutic target for NPC patients with cisplatin resistance.

Keywords: nasopharyngeal carcinoma, SLC1A6, cisplatin, radiation-resistance, glutamate

\section{INTRODUCTION}

Nasopharyngeal carcinoma (NPC) is a malignant tumor in the head and neck with high incidence in southern China and Southeast Asia (Chen et al., 2019; Ji et al., 2019). According to the guidelines of the National Comprehensive Cancer Network ( $\mathrm{NCCN})$, radiotherapy and cisplatin-based regimens are the main treatments for NPC patients (Pfister et al., 2020). The cisplatin-based concurrent chemoradiotherapy has been proven to improve the outcome of early and locally advanced NPC (Chen et al., 2011). However, there are a small portion of patients who did not response effectively or became recurrent to these treatments, and their prognosis remains poor (Chen et al., 2011; Karam et al., 2016).

The antitumor mechanism of cisplatin is to form covalent DNA adducts thus interfering with DNA repair (Wang and Lippard, 2005). The combination of cisplatin provides a synergetic effect for 
radiotherapy for the reason that cisplatin could enhance the sensitivity to radiation (Boeckman et al., 2005). Interestingly, some studies have revealed that cancer cells could acquire cisplatin resistance after radiation therapy (Eichholtz-Wirth, 1995; Zhuang et al., 2019). Based on our clinical observations, the phenomenon of cisplatin resistance is commonly seen in NPC patients who also resistant to radiotherapy. However, the association of cisplatin and radiation resistance is elusive.

The SLC1A6 (Solute Carrier Family 1 Member 6) is a member of the SLC1A family, which consists of the excitatory amino acid transporters EAAT1-EAAT5 (designated as SLC1A1-3, 6-7) and the alanine serine cysteine transporters ASCT1-ASCT2 (designated as SLC1A4-5) in mammals (Freidman et al., 2020). This transmembrane transporter encoded by SLC1A6 mediates the uptake of L-glutamate and L/D-aspartate (Fairman et al., 1995; Ryan et al., 2009; Vandenberg and Ryan, 2013; Guskov et al., 2016). Although the role of SLC1A6 in cancers was not well documented, other members of the SLC1A family were reported to be overexpressed in multiple tumors and predict poor prognosis. For example, expression of SLC1A1, SLC1A2 or SLC1A3 contributed to promoting tumor progression in solid tumors, such as lung cancer, glioma, and gastric cancer (Ye et al., 1999; de Groot et al., 2005; Tao et al., 2011; Xu et al., 2020; Guo et al., 2021). Besides, the SLC1A members were also reported to be associated with drug resistance. SLC1A1 was upregulated in oxaliplatin-resistant colorectal cancers (Pedraz-Cuesta et al., 2015), and SLC1A3 was associated with L-asparaginase resistance in acute lymphoblastic leukemia (Sun et al., 2019). As mammalian transporters of amino acids, members of the SLC1A family are implicated to impact drug-related metabolic profiles in tumor cells.

In the present study, we established two radioresistant NPC cell lines, HONE1-IR and CNE2-IR. We found that the radioresistant NPC cells acquired the characteristic of reduced cisplatin sensitivity, which was associated with the upregulation of SLC1A6. By inducing SLC1A6, HONE1-IR and CNE2-IR cells increased the cellular glutamate level and drug resistance genes, leading to reduced cisplatin sensitivity.

\section{MATERIALS AND METHODS}

\section{Ethical Statement}

The approval of this study was obtained from the Ethics Committee of Sun Yat-sen University Cancer Center (SYSUCC, Guangzhou, China). This study met the ethical standard of the Declaration of Helsinki. The nasopharyngeal biopsy was performed in all patients who have submitted their informed consent.

\section{Patients and Specimens}

A total of 78 NPC patients treated in Sun Yat-sen University Cancer Center were recruited and completely followed up from February 2011 to August 2014. The NPC biopsy specimens were fixed in $4 \%$ paraformaldehyde and paraffin-embedded and used for immunohistochemical (IHC) analysis of SLC1A6 expression.
SLC1A6 expression was scored according to the staining intensity and percentage of positively stained cells. The staining intensity score of SLC1A6 was graded as follows: 0, no staining; 1, weak staining (light yellow); 2, moderate staining (yellow-brown); 3 , intense staining (brown). The staining percentage score of SLC1A6 was graded as follows: 1, percentage of positive cells less than $30 \% ; 2$, percentage of positive cells between 30 and $60 \%$; 3 , percentage of positive cells more than $60 \%$. The total-score was calculated as the formula: Total score $=\sum$ (Intensity score $\times$ percentage score $)=(1 \times$ percentage score $)+(2 \times$ percentage score $)+(3 \times$ percentage score $)$. Finally, the intensity score, percentage score, and total score were used to verify the prognostic value of SLC1A6 expression for overall survival (OS).

\section{Immunohistochemistry}

The 5- $\mu \mathrm{m}$ paraffin sections were deparaffinized with xylene and rehydrated in graded ethanol. Antigen retrieval was achieved by placing the sections in sodium citrate buffer $\left(\mathrm{pH} \mathrm{6.0)}\right.$ at $95^{\circ} \mathrm{C}$ for 20 min. The sections were blocked with 5\% goat serum in PBS and incubated with primary antibody against SLC1A6 (1:100, Thermo fisher, the United States) overnight at $4^{\circ} \mathrm{C}$. The next day, the sections were stained with SP-9000 Detection Kits (BiotinStreptavidin HRP Detection Systems, ZSGB-Bio, China) and the DAB Kit (ZSGB-Bio, China) was used for color development according to the manufacturer's manual. The sections were counterstained with hematoxylin and observed with a light microscope.

\section{Cells and Cell Culture}

Human NPC cell lines CNE2 and HONE1 were gifted from Professor Chaonan Qian (SYSUCC) (Guo et al., 2020). Both the CNE2 and HONE1 cell lines, and their radioresistant cell lines HONE1-IR and CNE2-IR, were cultured in RPMI-1640 medium (Gibco, Thermo fisher, United States), supplemented with $10 \%$ fetal bovine serum (FBS, Gibco) and 1\% antibiotics (PenicillinStreptomycin). Another head and neck cell line SCC9 was cultured in F12-Dulbecco's modified Eagle's medium (Gibco, Thermo fisher, United States), supplemented with 10\% FBS and $1 \%$ antibiotics (Penicillin-Streptomycin). Customized RPMI-1640 (deprived glutamic acid or aspartic acid) was ordered from Weiga Biotechnology Company (Guangzhou, China), supplemented with $10 \%$ dialyzed FBS (Gibco, Thermo fisher, United States). All cells were incubated in a humidified atmosphere with $5 \% \mathrm{CO}_{2}$ at $37^{\circ} \mathrm{C}$.

\section{Establishment of Radioresistant Cell Lines}

According to our previous study (Guo et al., 2020), HONE1 cells were cultured in RPMI-1640 medium supplemented with $10 \%$ FBS and reached approximately $50 \%$ confluence in $25-\mathrm{cm}^{2}$ flasks. Cells were treated with a dose of $6 \mathrm{~Gy}$ radiation using an X-rays generator. After radiation, the culture medium was replaced with complete fresh medium and cells were returned to the incubator. Cells were passaged until they reached approximately $90 \%$ confluence. The fractionated irradiations were repeated five times and reached a total dose of $30 \mathrm{~Gy}$. The interval between each radiation was at least 2 -weeks for all cells. Radioresistant cell population were selected and were referred as HONE1-IR cells. 
The parental cells without irradiation were used as control cells. The CNE2-IR cells were kindly provided by Professor Yunfei Xia (SYSUCC).

\section{Quantitative Real-Time PCR}

The total RNAs were extracted by the TRIzol reagents (RNAiso PLUS, Invitrogen, US) following the manufacturer's manuals. The isolated RNAs were assessed by Bioanalyzer 2,200 (Agilent, Uunited States) to determine their concentrations and quality before conversion to cDNA. Quantitative RT-PCR was carried out using the Real-Time PCR Detection System (Lightcycler 480 II, Roche, United States) based on the manufacturer's manual. Values were expressed as fold changes of the controls using the $2 \mathrm{e}^{-\Delta \Delta \mathrm{Ct}}$ method.

\section{RNA-Seq Transcriptome Analysis}

NPC Cells (HONE1 and HONE1-IR, CNE2, and CNE2-IR) were cultured and their total RNAs were extracted as described above and kept at $-80^{\circ} \mathrm{C}$. Sequencing and bioinformatic analysis were performed by DESeq 2 and edgeR platforms with the aid of Novogene Company (Beijing, China). The intensity was used to generate the heatmap by Novomagic platform (Novogene Company). Differentially expressed genes were determined when $p<0.05$ and the absolute log2 fold change of expression was greater than 3 .

\section{MTS Assay}

HONE1 (HONE1-IR and NC cells) and CNE2 (CNE2-IR and NC cells) were planted into 96 -well plates at $1-2 \times 10^{3}$ cells/200 $\mu \mathrm{l} /$ well, treated with increasing concentration of cisplatin for $24 \mathrm{~h}$. In the last $2 \mathrm{~h}$ of incubation, $20 \mu \mathrm{L}$ of MTS tetrazolium (Promega, United States) was added to each well based on the manufacturer's protocols. Cell viability was examined by assessing the light absorbance at 490-500 $\mathrm{nm}$. The cell survival curves were drawn based on the results obtained.

\section{Colony Formation}

HONE1 (HONE1-IR cells and NC cells) and CNE2 (CNE2-IR and NC cells) were planted into 6 -well plates at $5 \times 10^{2}$ cells/well. Subsequently, cisplatin was added to the cultured medium at the concentration of $20 \mu \mathrm{M}$ for $24 \mathrm{~h}$ of treatment. After that, the culture medium was replaced by a fresh medium. The cells were cultured for 2 weeks. Crystal violet was used to stain the colonies.

\section{Small Interfering RNA Transfection}

HONE1-IR and CNE2-IR cells were planted into 6-well plates one day before transfection to reach about $60-70 \%$ confluence. The siRNAs targeting SLC1A6 gene were purchased from Ruibo Company (Guangzhou, China). Transient transfection of HONE1-IR and CNE2-IR cells was performed using Lipofectamine RNAiMax (Invitrogen, United States) according to the manufacturer's protocols. Cells were transfected with a total of 50 pmol siRNA and subjected to Western blot and MTS assays after $24-48 \mathrm{~h}$ post-transfection. The siRNA sequences for SLC1A6 were as follows:

SLC1A6-siRNA-1 : $5^{\prime}$-AUGAAAACUGCAAUGACUGUA-3'

SLC1A6-siRNA-2 : 5' -AAGGAAUAAGCCAACGAUGAC-3'

\section{Establishment of SLC1A6 Overexpressed Stable Cell Lines}

The cDNA of SLC1A6 was synthesized according to the human full-length open reading frame of SLC1A6 mRNA (NM_001,272,087) and integrated into pcDNA3.1 plasmid. The lentiviral expression plasmid was used to transfect 293T cells for packaging. The culture media of transfected $293 \mathrm{~T}$ cells was harvested and used to transfect HONE1 or CNE2 cells. Cells expressing SLC1A6 were selected with puromycin (Sigma-Aldrich, United States) in the concentration of $2 \mu \mathrm{g} / \mathrm{ml}$. SLC1A6-overexpressed stable cells were established after 10 days selection.

\section{Western Blotting Analysis}

Following treatments, cells were lyzed by RIPA buffer (Beyotime, China) containing protease and phosphatase inhibitors (Life Technologies, United States). Protein concentrations were detected by a BCA kit (Thermo Fisher, United States). Proteins of samples were subjected to $8-10 \%$ SDS-PAGE and transferred to PVDF membranes (Biorad, United States). After being blocked with 5\% non-fat milk for $1 \mathrm{~h}$, membranes were incubated with primary antibodies at $4^{\circ} \mathrm{C}$ overnight. Primary antibodies against SLC1A6 (1:1,000, Thermo fisher), $\gamma \mathrm{H} 2 \mathrm{AX}$ (1:1,000, Cell Signaling), Beta-actin (1:1,000, Cell Signaling) were used. Then the membranes were washed with TBS containing $0.1 \%$ Tween-20, followed by incubation with HRP anti-rabbit (1:1,000, Cell Signaling) secondary antibody. The band intensity values were normalized to that of Beta-actin.

\section{Measurement of Glutamate or Aspartate Level}

A total of $10 \times 10^{6}$ cells were collected and lyzed by ultrasonication (low frequency, 3s, 20 times), glutamate or aspartate content was extracted by glutamate assay kit (Solarbio, China) or aspartate assay kit (Abnova, Taiwan). The level of glutamate or aspartate was detected by comparing the light absorbance value with the standard solution curve. The absorbance value was measured at $340 \mathrm{~nm}$ or $570 \mathrm{~nm}$, respectively.

\section{Online and Public Database}

The correlation of SLC1A6 expression and survival outcome in patients with head and neck squamous cell carcinoma (HNSCC) in the TCGA database was analyzed on the GEPIA website (http://gepia.cancer-pku.cn/).

\section{Statistical Analysis}

The data were expressed as the mean \pm SD (the standard deviation). Comparisons between two groups were analyzed by unpaired Student's $t$ test. Comparisons among groups were performed by one-way analysis of variance (ANOVA) followed by Tukey's test. Significant $p$ value was considered as $<0.05$. In all cases, ${ }^{\star} p<0.05,{ }^{*} p<<$ $0.01,{ }^{* *} p<0.001$. 

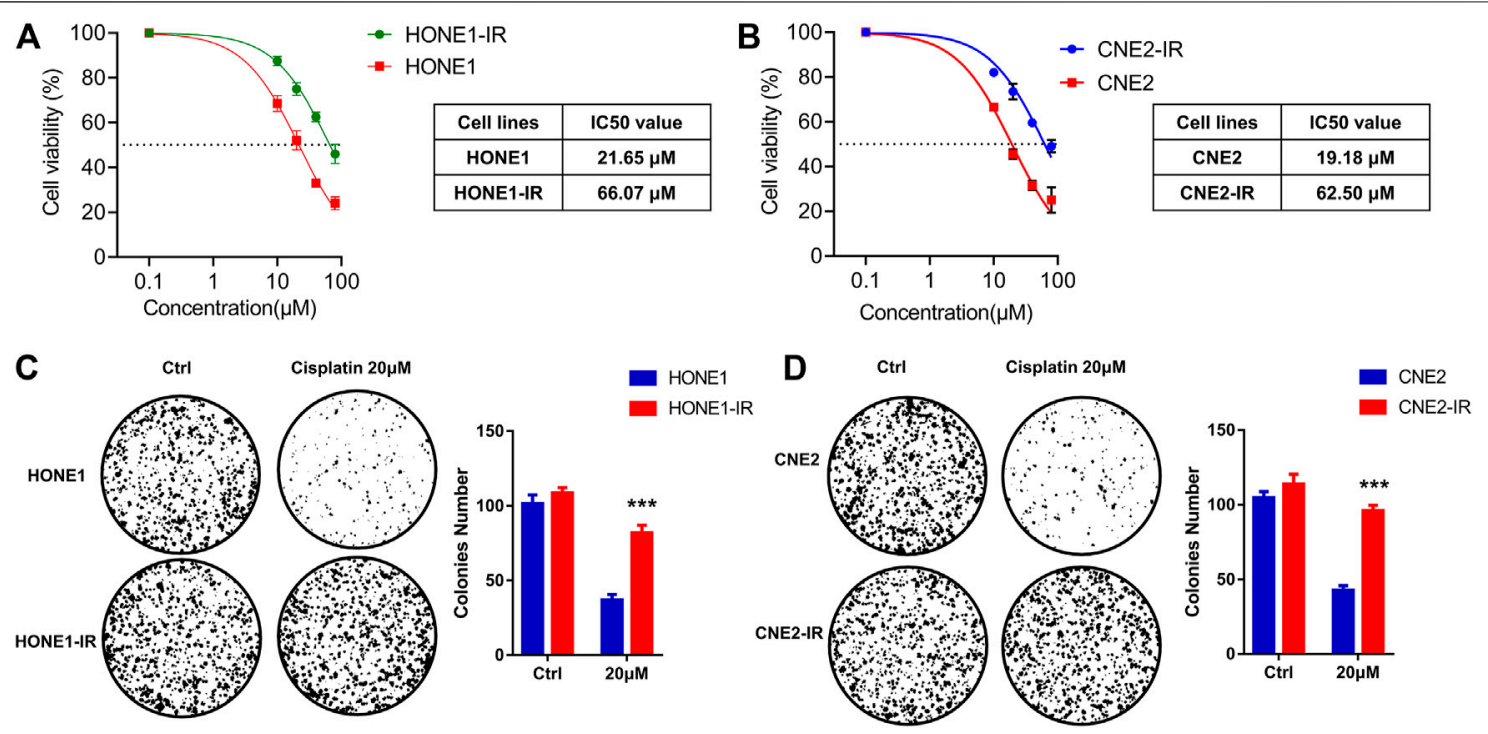

FIGURE 1 | Radioresistant HONE1-IR and CNE2-IR cells showed reduced sensitivity to cisplatin. (A-B) MTS assay on the IC50 values of radioresistant NPC cell lines HONE1-IR and CNE2-IR, as well as their parental cell lines HONE1 and CNE2 with cisplatin treatment. ANOVA test (C-D) Colony formation assay on the radioresistant NPC cell lines and their parental cell lines. Unpaired Student's t test. ${ }^{*} p<0.05,{ }^{\star *} p<0.01,{ }^{* \star *} p<0.001$.

\section{RESULTS}

\section{Radioresistant HONE1-IR and CNE2-IR Cells Showed Reduced Sensitivity to Cisplatin}

Two radioresistant human NPC cell lines, HONE1-IR and CNE2IR, were generated and their resistance to radiation was verified by survival assay (Guo et al., 2020). As the sensitivity to cisplatin is closely associated with radiotherapy efficacy, we compared the sensitivity to cisplatin in radioresistant NPC and their parental cells. MTS assay supported that radioresistant NPC cells exhibited reduced sensitivity to cisplatin compared to their parental cells. Subsequent analysis of chemosensitivity indicated that the cisplatin IC50 of HONE1-IR cells was significantly higher than that of their parental cells (66.07 vs. $21.65 \mu \mathrm{M})$ ). Similarly, CNE2-IR cells also showed higher IC50 than that of their parental cells $(62.50$ vs. $19.18 \mu \mathrm{M})$ (Figures 1A,B). Colony formation assay showed that cisplatin treatment significantly reduced tumor cell proliferation in HONE1 and CNE2 cells, but not in HONE1-IR and CNE2-IR cells (Figures 1C,D). These findings indicated that radioresistant NPC cells reduced cisplatin sensitivity.

\section{SLC1A6 Gene was Up-Regulated in Both HONE1-IR and CNE2-IR Cells}

To explore the potential regulators of cisplatin sensitivity in radioresistant NPC cells, RNA-Sequence was performed to compare the transcriptome profile of HONE1-IR cells and their parental cells. The cutoff criteria were more than 3-fold or less than 3-fold for upregulation or downregulation, respectively. As for CHE2-IR cells and their parental cells, the same method was performed. Heatmap showed a total of 573 genes in HONE1 cells (HONE1-IR cells vs. their parental cells) and 404 genes in CNE2 cells (CNE2-IR cells vs. their parental cells) were identified to be differentially expressed (Figures 2A,B). There were 30 common genes upregulated in both HONE1-IR and CNE2-IR cells compared to their parental cells (Figure 2C). SLC1A6 gene was particularly noted in the top nine over-expressed genes (Figure 2D) and verified by qRTPCR (Figure 2E). These results indicated that SLC1A6 might be a crucial gene associated with cisplatin or radiation sensitivity in radioresistant NPC cells.

\section{SLC1A6 Overexpression Conferred Reduced Cisplatin and Radiation Sensitivity in Radioresistant NPC Cells}

Furthermore, the up-regulation of SLC1A6 has been verified by Western blots in both HONE1-IR and CNE2-IR cells compared to their parental cells (Figure 3A). To further investigate the role of SLC1A6 in NPC radioresistant cells, siRNA or SLC1A6 overexpression lentivirus was used to knock down or up regulate the expression of SLC1A6 in cells (Figure 3B). Results showed that downregulation of SLC1A6 gene resensitized radioresistant NPC cells to cisplatin treatment (Figure 3C). Moreover, overexpression of SLC1A6 decreased the sensitivity to cisplatin in parental cells (Figure 3D). Besides, modulation of the SLC1A6 also impacted the sensitivity to radiation in radioresistant NPC and parental cells (Figures 3E,F). Cisplatin and radiation both cause DNA damage. Increased gamma-H2AX ( $\gamma \mathrm{H} 2 \mathrm{AX})$ expression, a biomarker of DNA damage, was noticed in radioresistant NPC cells by 


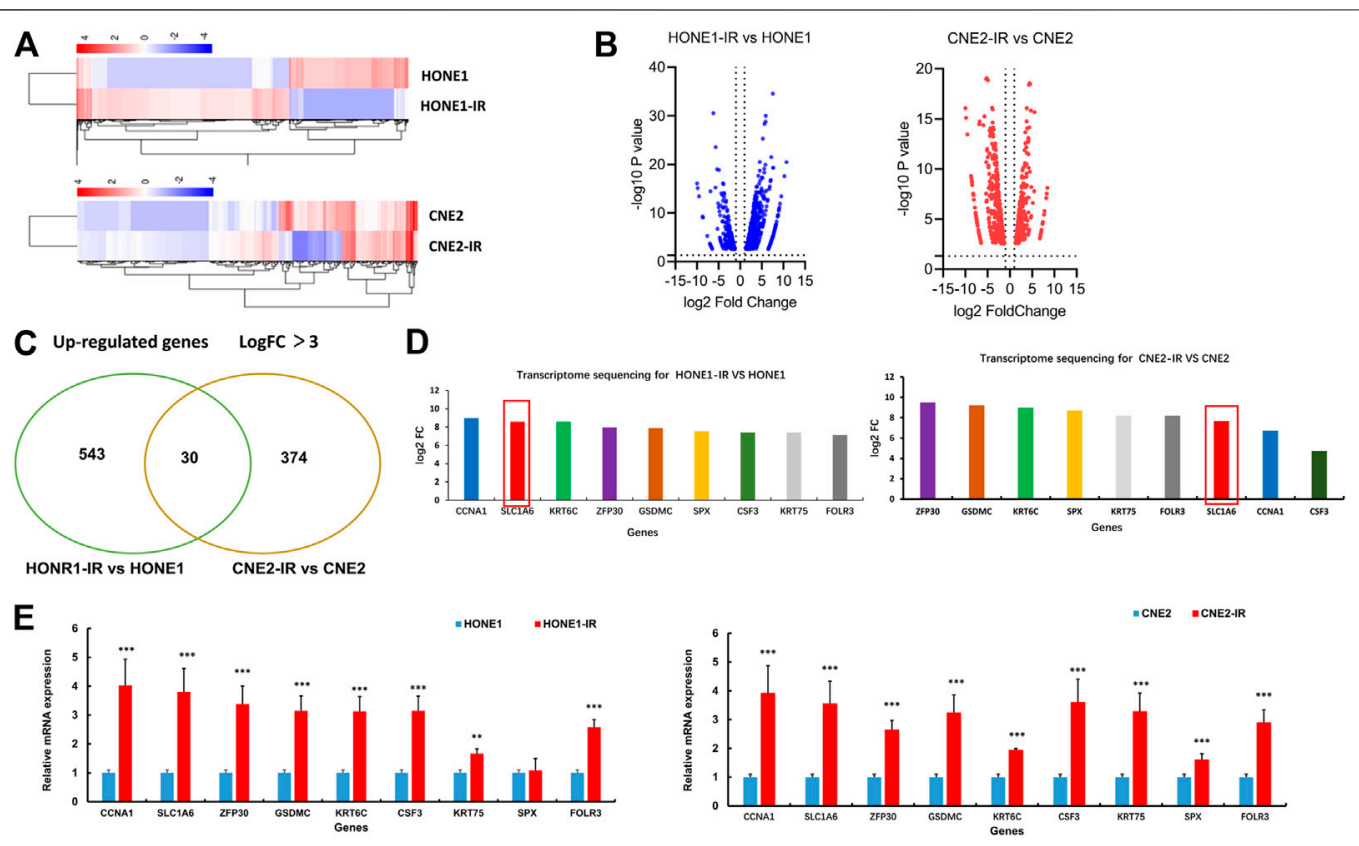

FIGURE 2 | SLC1A6 up-regulated in radioresistant NPC cells. (A) Heat maps showing the expression pattern of up-regulated and down-regulated genes in HONE1 cells (HONE1-IR vs. HONE1) and CNE2 cells (CNE2-IR vs. CNE2). Red or blue represents high or low expression, respectively (Raw Z score). (B) Volcano maps showing the expression pattern of genes. (C) Venn maps showing 573 up-regulated genes in HONE1R vs. HONE1 cells and 404 up-regulated genes in CNE2-IR vs. CNE2 cells, with 30 common genes. (D) Transcriptome sequencing showing the highest fold change in up-regulated nine genes, co-expressed in both radioresistant NPC cells, including SLC1A6. (E) qRT-PCR verified the different mRNA expression of the nine genes. Unpaired Student's $t$ test. ${ }^{\star} p<0.05$, ${ }^{\star \star} p<0.01$, ${ }^{\star \star *} p<0.001$.

knocking down SLC1A6, followed by cisplatin or radiation treatment (Figures 3G,H). These results demonstrated that SLC1A6 contributed to reducing cisplatin and radiation sensitivity of radioresistant NPC cells.

\section{SLC1A6 Induced by Radiation Treatment and Correlated with Poor Prognosis}

As SLC1A6 gene was up-regulated in the radioresistant NPC cells, we next investigated the impact of radiation on SLC1A6. We observed both RNA (Figure 4A) and protein levels (Figure 4B) of SLC1A6 increased during radiation treatment in NPC cells. As NPC belongs to HNSCC, another HNSCC cell line SCC9, was utilized to further verify the role of the SLC1A6 gene on the sensitivity to cisplatin. Similarly, SLC1A6 up-regulation was seen during radiation treatment in SCC9 cells (Figures 4A,B). SLC1A6 over-expressed SCC9 cells exhibited low sensitivity to cisplatin (Figure 4C). Analysis on TCGA database showed that high SLC1A6 expression was correlated with poor prognosis in HNSCC patients (Figure 4D). To further validate the prognostic role of the SLC1A6 in NPC patients, we collected 78 biopsies from NPC patients in Sun Yat-sen University Cancer Center. We demonstrated that high SLC1A6 expression was correlated with poor prognosis in these patients (Figures 4E,F). These results demonstrated that overexpression of SLC1A6 was associated with low therapeutic efficacy and poor survival in NPC patients.

\section{SLC1A6 Up-Regulated Glutamate Level and Drug Resistance Genes}

Further experiments were conducted to elucidate the underlying mechanism of SLC1A6 in regulating cisplatin sensitivity in radioresistant NPC cells. SLC1A6 is one of the members of the EAATs family, which transport aspartate, glutamate, and cysteine. These amino acids serve as substrates in several biochemical and metabolic pathways in cancer cells. Previous studies have reported that the EAATs-mediated therapeutic resistance is related to altered tumor metabolic profiles ( $\mathrm{Ye}$ et al., 1999; de Groot et al., 2005; Tao et al., 2011; PedrazCuesta et al., 2015; Sun et al., 2019; Xu et al., 2020; Guo et al., 2021). It was found that the level of glutamate and aspartate increased in the radioresistant NPC cells compared to their parental cells (Figure 5A). In addition, deprivation of glutamate, not aspartate, in the culture medium re-sensitized radioresistant NPC cells to cisplatin treatment (Figure 5B). Moreover, PCR analysis revealed that the expression of CYP1A1, CYP2C8, CYP2D6, DHFR, GSTP1, and SULT1E1 genes, that are associated with drug catabolism, were significantly higher in radioresistant NPC cells compared to their parental cells (Figure 5C). ABCC1 and ABCC3, that are associated with drug transportation, were also elevated in radioresistant NPC cells (Figure 5C). The expressions of these genes were significantly decreased when SLC1A6 was knocked down in radioresistant NPC cells (Figure 5D). These results collectively supported that SLC1A6 overexpression reduced 

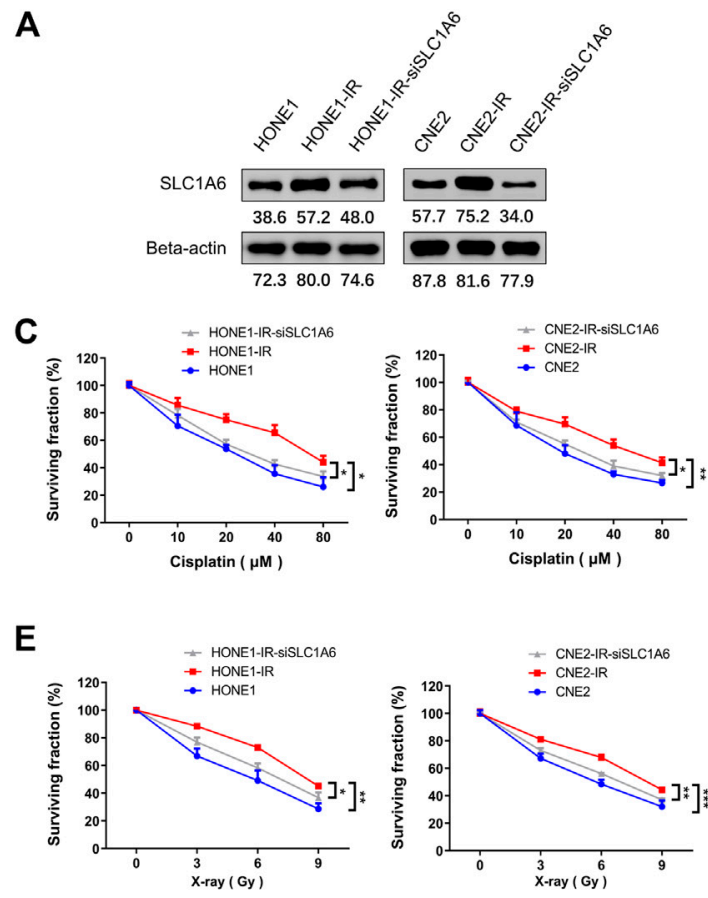

$\mathbf{G}$
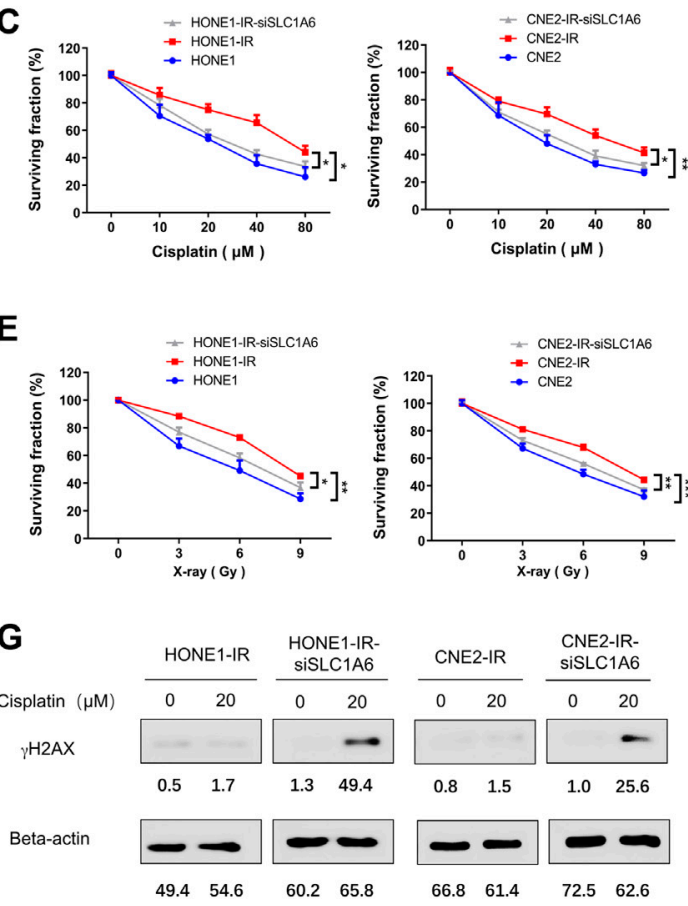
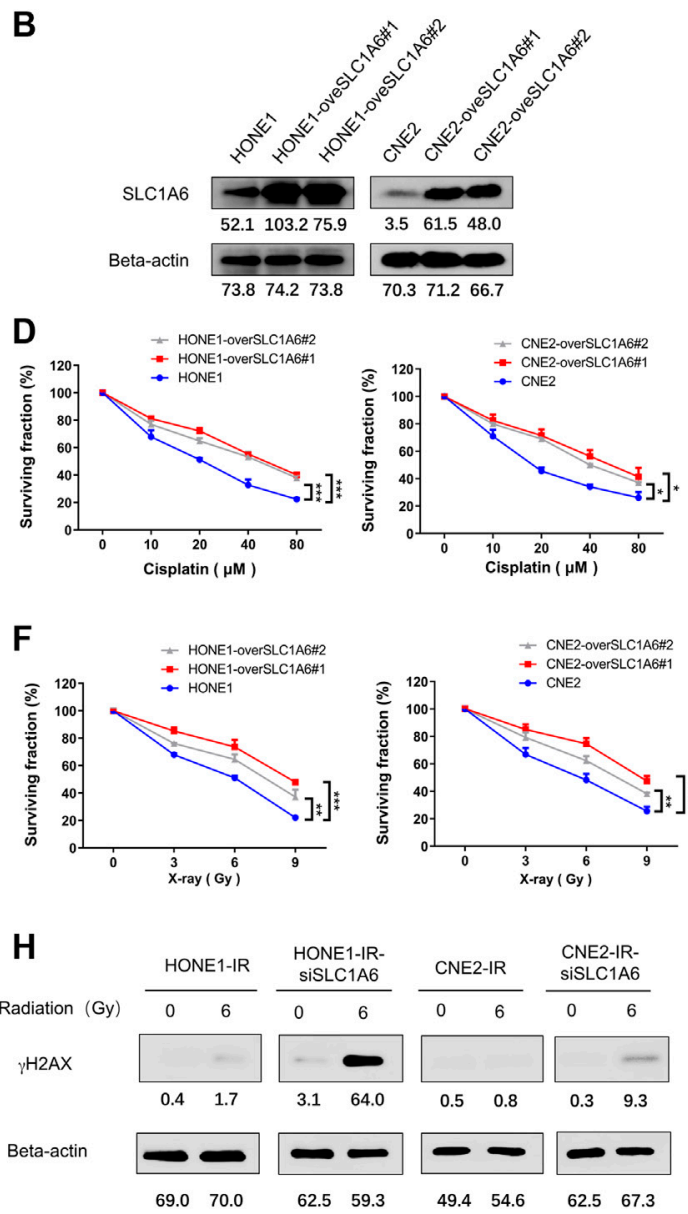

FIGURE 3 | SLC1A6 mediated reduced sensitivity to cisplatin and radiation in radioresistant NPC cells. (A) SLC1A6 and Beta-actin protein expression of HONE1, CNE2, HONE1-IR, CNE2-IR and radioresistant NPC SLC1A6 knockdown cell lines, detected by Western blots. (B) SLC1A6 and Beta-actin protein expression of HONE1, CNE2, HONE1-overSLC1A6 and CNE2-overSLC1A6 cell lines, detected by Western blots. (C-D) MTS assays conducted in HONE1, CNE2, HONE1-IR, CNE2-IR, radioresistant NPC SLC1A6 knockdown cell lines or SLC1A6 overexpressed cell lines, treated with cisplatin. The difference was analyzed by repeated ANOVA. (E-F) MTS assays conducted in HONE1, CNE2, HONE1-IR, CNE2-IR, radioresistant NPC SLC1A6 knockdown cell lines or SLC1A6 overexpressed cell lines, treated with fractional radiation. The difference was analyzed by repeated ANOVA. (G-H) The levels of $\gamma \mathrm{H} 2 \mathrm{AX}$ and Beta-actin analyzed by Western blots. ${ }^{\star} p<0.05,{ }^{\star \star} p<0.01,{ }^{\star \star \star} p<0.001$.

cisplatin sensitivity in radioresistant NPC cells by increasing the level of glutamate and drug resistance genes.

\section{DISCUSSION}

Radiation resistance has been a significant obstacle for the local control of NPC. Some patients who are resistant to radiotherapy are also not sensitive to cisplatin treatment. In this study, we observed the decreased sensitivity to cisplatin in radiation resistant NPC cells. Although previous studies have revealed the mechanism of radiationresistance or cisplatin resistance, the relationship between radiation and cisplatin resistance is complicated and not elucidated. The cause of cisplatin resistance, included aberrant repair of DNA damage, apoptosis pathway defects, activation of drug export system, altered cellular metabolism, reduced oxidative stress, and cancer stem cell induction, etc. (Ikuta et al., 2005; Galluzzi et al., 2012; Zhang et al., 2012; Cruz-Bermúdez et al., 2019) As both radiation and cisplatin cause DNA damage in tumor cells, they might share the same biological pathway to reverse DNA damage. Cross-resistance mechanisms reported by studies include elevated GSH level, DNA repair enzymes, NFkB and TNFa, etc. (Chao et al., 1991; Poppenborg et al., 1997; Zhu et al., 2019)

Here, the identification of SLC1A6 as the crucial gene conferring reduced cisplatin sensitivity in radiation-resistant NPC cells is novel. In this study, it was found that SLC1A6 expression was upregulated in HONE1-IR and CNE2-IR cells. Down-regulating SLC1A6 expression could significantly rescue the cisplatin sensitivity in HONE1-IR and CNE2-IR cells. The SLC1A6 could be the common factor to reduce DNA damage from radiation or cisplatin treatment, which was confirmed by our study. Our results were in consistent with previous studies that described the radiation-induced cisplatin resistance (Eichholtz-Wirth et al., 1993; Eichholtz-Wirth, 1995; Zhuang et al., 2019). The cisplatin resistance acquired during radiation could explain the phenomenon that some patients with radio-resistance also didn't respond to cisplatin 


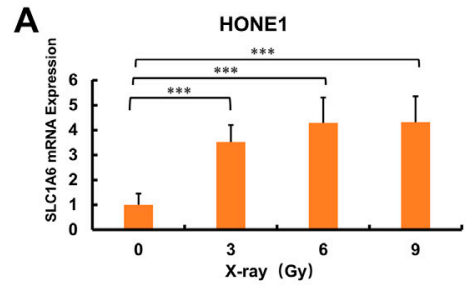

B

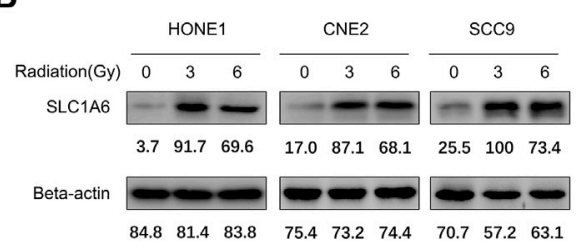

E
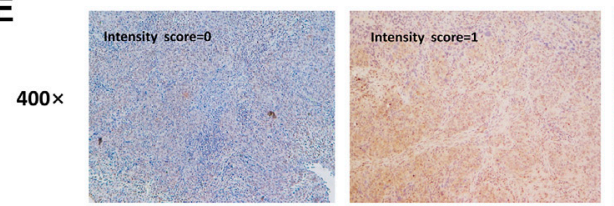

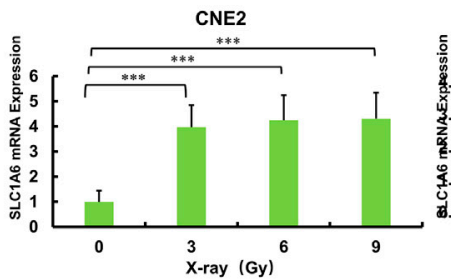

C

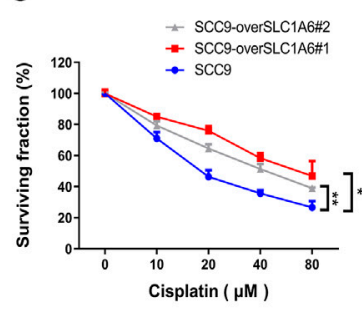

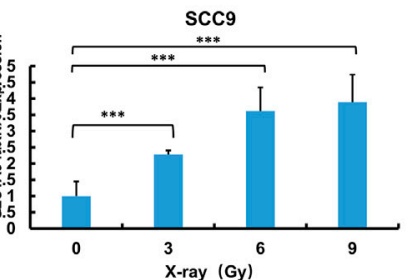

D

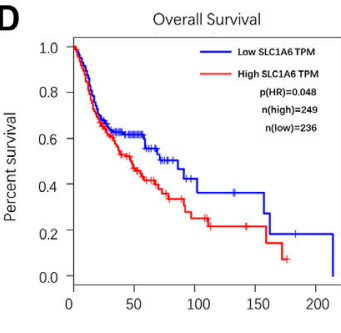

F
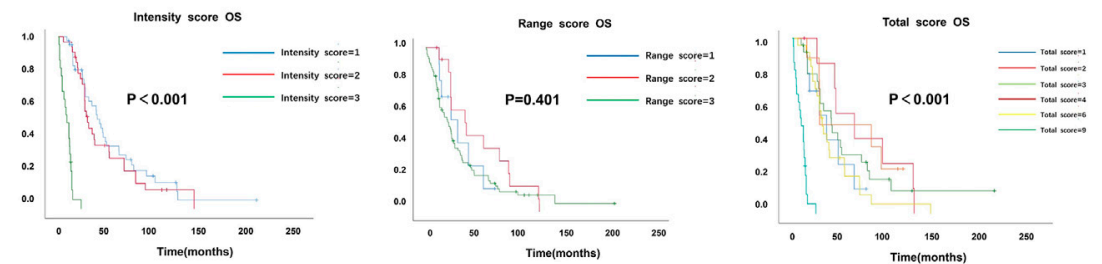

FIGURE 4 | SLC1A6 induced by radiation treatment and correlated with poor prognosis. (A) The mRNA expression of SLC1A6 in HONE1, CNE2, and SCC9 cells treated with increasing radiation doses. (B) The protein expression of SLC1A6 in HONE1, CNE2, and SCC9 cells treated with increasing doses of radiation. (C) Cell viability examined by MTS assays in SCC9 cells treated with cisplatin. (D) Patients with SLC1A6 overexpression showed poor overall survival analyzed with GEPIA websites. (E) Representative micrographs (400x). All images were acquired and processed at identical conditions. (F) OS curves on the SLC1A6 expression in validation set consisting of 78 patients with NPC from Sun Yat-sen University Cancer Center, using intensity score, percentage score, or total score, respectively. The differences were measured by Kaplan-Meier log-rank test. ${ }^{\star} p<0.05,{ }^{* *} p<0.01,{ }^{\star \star *} p<0.001$.

treatment and correlated with the radiation-induced SLC1A6 upregulation. However, the mechanism of radiation induced SLC1A6 overexpression remained far from understood. We hypothesized that genetic reprogramming happened in the process of DNA injury and repair upon radiation.

SLC1A family is thought to contribute to tumor progression by regulating microenvironments and metabolic profiles (Ye et al., 1999; de Groot et al., 2005; Tao et al., 2011; Pedraz-Cuesta et al., 2015; Sun et al., 2019; Freidman et al., 2020; Xu et al., 2020; Guo et al., 2021). SLC1A6 transports aspartate, glutamate, and cysteine, and regulation of these amino acids is essential for numerous biochemical and metabolic pathways such as the TCA cycle or nucleotide synthesis. For example, glutamate could be transformed into glutamine, facilitating nucleotide synthesis and repair DNA damage (Fu et al., 2019). The endocrine resistance breast cancer cells would increase aspartate and glutamate import to sustain DNA, lipid, and protein synthesis (Bacci et al., 2019). We found both glutamate and aspartate were elevated in radioresistant NPC cells. However, only glutamate played a vital role in resisting DNA injury from cisplatin treatment. These findings suggested that SLC1A6 contributed to metabolic reprogramming in radioresistant NPC cells.

We also uncovered that SLC1A6 promoted the upregulation of drug catabolic genes (CYP1A1, CYP2C8, CYP2D6, DHFR, GSTP1, and SULT1E1) and drug transport genes (ABCC1 and ABCC3). The CYP enzymes have been extensively investigated in drug metabolism, and their inhibitors were proven to be effective in reversing cisplatin sensitivity in cancer cells (Sonawane et al., 2019). Gstp1, a GST family member, is involved in the detoxification of cisplatin via cisplatin-glutathione adducts formation (Li et al., 2019). DHFR and SULT1E1 have been reported to be up-regulated in cisplatin-resistant cells (Marverti et al., 2009; Varamo et al., 2019). The ABC family of transporters is referred to as multidrug resistance proteins that transport substrates across the cellular membranes (Chen et al., 2016). These results implicated that SLC1A6 contributed to cisplatin resistance in radioresistant NPC cells through multiple factors. 

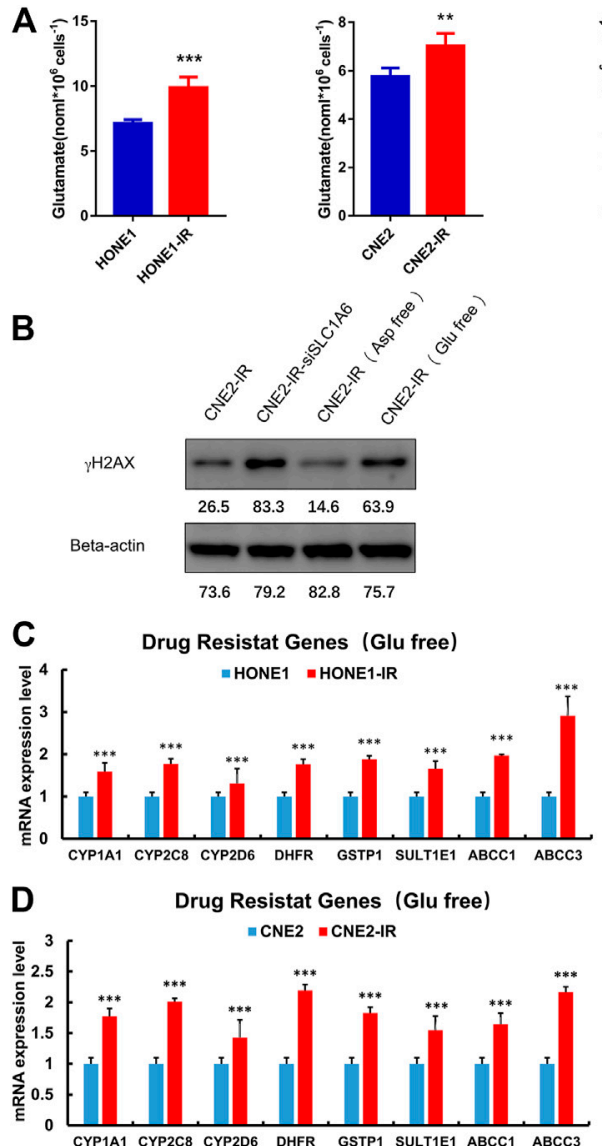
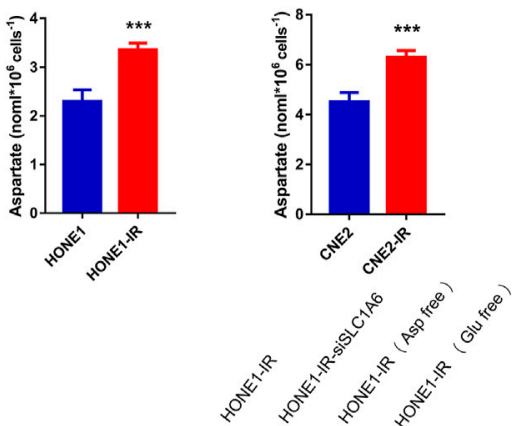

$\gamma \mathrm{H} 2 \mathrm{AX}$

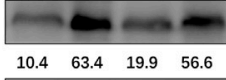

Beta-actin

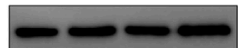

$68.9 \quad 78.9-70.8 \quad 759$

Drug Resistat Genes (Glu free)

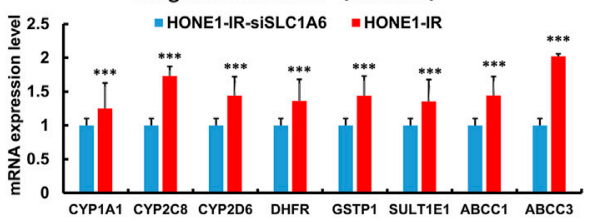

Drug Resistat Genes (Glu free)

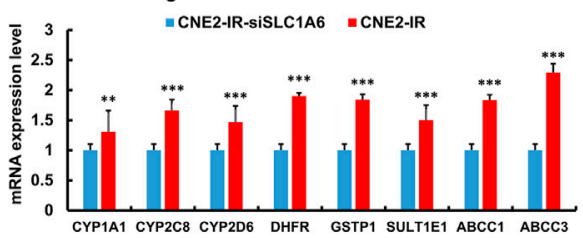

FIGURE 5 | SLC1A6 increased glutamate level and drug resistance gene expression. (A) The level of glutamate and aspartate in radioresistant NPC cells and their parental cells. (B) The level of $\gamma \mathrm{H} 2 \mathrm{AX}$ and Beta-actin in radioresistant NPC cells, radioresistant NPC SLC1A6 knockdown cells, radioresistant NPC cells cultured with glutamic acid free medium and radioresistant NPC cells cultured with aspartic acid free medium, treated with cisplatin. (C-D) RT-PCR on the mRNA expression of drug resistance genes in NPC cells, radioresistant NPC cells and radioresistant SLC1A6 knockdown cells. Unpaired Student's t test. ${ }^{\star} p<0.05$, ${ }^{\star \star} p<0.01,{ }^{\star \star \star} p<0.001$.

In summary, we identified the upregulation of SLC1A6 in radioresistant NPC cells. Overexpression of SLC1A6 is correlated with reduced sensitivity to cisplatin by elevating the level of glutamate and drug resistance genes. Targeting SLC1A6 could be a potential strategy to enhance cisplatin sensitivity in NPC patients.

\section{DATA AVAILABILITY STATEMENT}

The datasets presented in this study can be found in online repositories. The names of the repository/repositories and accession number(s) can be found below: NCBI SRA, PRJNA700383.

\section{ETHICS STATEMENT}

This study was approved by the Ethics Committee of the Sun Yat-sen University Cancer Center, and met the ethical standard of the Declaration of Helsinki. Informed consent was obtained from each patient before obtaining the nasopharyngeal biopsy.

\section{AUTHOR CONTRIBUTIONS}

WH, LW, LC, JY, JW and LN conducted experiments and acquired the data; WH, LW, LC and JY analyzed the data; WH, LW and LC wrote the paper; YC and XL conceived and designed the study. All authors read and agreed on the final manuscript.

\section{FUNDING}

This study was supported by National Natural Science Foundation of China (81902928, 81702762), Natural Science Foundation of Guangdong Province (2021A1515010602), Beijing Xisike Clinical Oncology Research Foundation (Y-MX2016-062). 


\section{REFERENCES}

Bacci, M., Lorito, N., Ippolito, L., Ramazzotti, M., Luti, S., Romagnoli, S., et al. (2019). Reprogramming of amino acid transporters to support aspartate and glutamate dependency sustains endocrine resistance in breast cancer. Cel Rep. 28, 104-118. doi:10.1016/j.celrep.2019.06.010

Boeckman, H. J., Trego, K. S., and Turchi, J. J. (2005). Cisplatin sensitizes cancer cells to ionizing radiation via inhibition of nonhomologous end joining. Mol. Cancer Res. 3, 277-285. doi:10.1158/1541-7786.MCR04-0032

Chao, C. C., Huang, S. L., Huang, H. M., and Lin-Chao, S. (1991). Cross-resistance to UV radiation of a cisplatin-resistant human cell line: overexpression of cellular factors that recognize UV-modified DNA. Mol. Cel. Biol. 11, 2075-2080. doi:10.1128/mcb.11.4.2075

Chen, Q.-Y., Wen, Y.-F., Guo, L., Liu, H., Huang, P.-Y., Mo, H.-Y., et al. (2011). Concurrent chemoradiotherapy vs radiotherapy alone in stage II nasopharyngeal carcinoma: phase III randomized trial. J. Natl. Cancer Inst. 103, 1761-1770. doi:10.1093/jnci/djr432

Chen, Y.-P., Chan, A. T. C., Le, Q.-T., Blanchard, P., Sun, Y., and Ma, J. (2019). Nasopharyngeal carcinoma. The Lancet 394, 64-80. doi:10.1016/S01406736(19)30956-0

Chen, Z., Shi, T., Zhang, L., Zhu, P., Deng, M., Huang, C., et al. (2016). Mammalian drug efflux transporters of the ATP binding cassette (ABC) family in multidrug resistance: a review of the past decade. Cancer Lett. 370, 153-164. doi:10.1016/j. canlet.2015.10.010

Cruz-Bermúdez, A., Laza-Briviesca, R., Vicente-Blanco, R. J., García-Grande, A., Coronado, M. J., Laine-Menéndez, S., et al. (2019). Cisplatin resistance involves a metabolic reprogramming through ROS and PGC-1a in NSCLC which can be overcome by OXPHOS inhibition. Free Radic. Biol. Med. 135, 167-181. doi:10. 1016/j.freeradbiomed.2019.03.009

de Groot, J. F., Liu, T. J., Fuller, G., and Yung, W. K. A. (2005). The excitatory amino acid transporter-2 induces apoptosis and decreases glioma growth in vitro and in vivo. Cancer Res. 65, 1934-1940. doi:10.1158/0008-5472. CAN-04-3626

Eichholtz-Wirth, H., Reidel, G., and Hietel, B. (1993). Radiation-induced transient cisplatin resistance in murine fibrosarcoma cells associated with elevated metallothionein content. Br. J. Cancer 67, 1001-1006. doi:10. 1038/bjc. 1993.183

Eichholtz-Wirth, H. (1995). Reversal of radiation-induced cisplatin resistance in murine fibrosarcoma cells by selective modulation of the cyclic GMPdependent transduction pathway. Br. J. Cancer 72, 287-292. doi:10.1038/bjc. 1995.326

Fairman, W. A., Vandenberg, R. J., Arriza, J. L., Kavanaught, M. P., and Amara, S. G. (1995). An excitatory amino-acid transporter with properties of a ligand-gated chloride channel. Nature 375, 599-603. doi:10.1038/ $375599 \mathrm{a} 0$

Freidman, N., Chen, I., Wu, Q., Briot, C., Holst, J., Font, J., et al. (2020). Amino acid transporters and exchangers from the SLC1A family: structure, mechanism and roles in physiology and cancer. Neurochem. Res. 45, 1268-1286. doi:10.1007/ s11064-019-02934-x

Fu, S., Li, Z., Xiao, L., Hu, W., Zhang, L., Xie, B., et al. (2019). Glutamine synthetase promotes radiation resistance via facilitating nucleotide metabolism and subsequent DNA damage repair. Cel Rep. 28, 1136-1143. doi:10.1016/j. celrep.2019.07.002

Galluzzi, L., Senovilla, L., Vitale, I., Michels, J., Martins, I., Kepp, O., et al. (2012). Molecular mechanisms of cisplatin resistance. Oncogene 31, 1869-1883. doi:10. 1038/onc. 2011.384

Guo, S.-S., Liu, R., Wen, Y.-F., Liu, L.-T., Yuan, L., Li, Y.-X., et al. (2020). Endogenous production of C-C motif chemokine ligand 2 by nasopharyngeal carcinoma cells drives radioresistance-associated metastasis. Cancer Lett. 468, 27-40. doi:10.1016/j.canlet.2019.10.008

Guo, W., Li, K., Sun, B., Xu, D., Tong, L., Yin, H., et al. (2021). Dysregulated glutamate transporter SLC1A1 propels cystine uptake via xc- for glutathione synthesis in lung cancer. Cancer Res. 81, 552-566. doi:10.1158/0008-5472. CAN-20-0617

Guskov, A., Jensen, S., Faustino, I., Marrink, S. J., and Slotboom, D. J. (2016). Coupled binding mechanism of three sodium ions and aspartate in the glutamate transporter homologue GltTk. Nat. Commun. 7, 13420. doi:10. 1038 /ncomms 13420

Ikuta, K., Takemura, K., Kihara, M., Naito, S., Lee, E., Shimizu, E., et al. (2005). Defects in apoptotic signal transduction in cisplatin-resistant non-small cell lung cancer cells. Oncol. Rep. 13, 1229-1234.

Ji, M. F., Sheng, W., Cheng, W. M., Ng, M. H., Wu, B. H., Yu, X., et al. (2019). Incidence and mortality of nasopharyngeal carcinoma: interim analysis of a cluster randomized controlled screening trial (PRO-NPC001) in southern China. Ann. Oncol. 30, 1630-1637. doi:10.1093/ annonc/mdz231

Karam, I., Huang, S. H., McNiven, A., Su, J., Xu, W., Waldron, J., et al. (2016) Outcomes after reirradiation for recurrent nasopharyngeal carcinoma: north American experience. Head Neck 38 (Suppl. 1), E1102-E1109. doi:10.1002/hed. 24166

Li, J., Ye, T., Liu, Y., Kong, L., Sun, Z., Liu, D., et al. (2019). Transcriptional activation of gstp1 by MEK/ERK signaling confers Chemo-Resistance to cisplatin in lung cancer stem cells. Front. Oncol. 9, 476. doi:10.3389/fonc. 2019.00476

Marverti, G., Ligabue, A., Paglietti, G., Corona, P., Piras, S., Vitale, G., et al. (2009). Collateral sensitivity to novel thymidylate synthase inhibitors correlates with folate cycle enzymes impairment in cisplatin-resistant human ovarian cancer cells. Eur. J. Pharmacol. 615, 17-26. doi:10. 1016/j.ejphar.2009.04.062

Pedraz-Cuesta, E., Christensen, S., Jensen, A. A., Jensen, N. F., Bunch, L., Romer, M. U., et al. (2015). The glutamate transport inhibitor DL-Threo$\beta$-Benzyloxyaspartic acid (DL-TBOA) differentially affects SN38- and oxaliplatin-induced death of drug-resistant colorectal cancer cells. BMC Cancer 15, 411. doi:10.1186/s12885-015-1405-8

Pfister, D. G., Spencer, S., Adelstein, D., Adkins, D., Anzai, Y., Brizel, D. M., et al. (2020). Head and neck cancers, version 2.2020, NCCN clinical practice guidelines in Oncology. J. Natl. Compr. Canc Netw. 18, 873-898. doi:10. 6004/jnccn.2020.0031

Poppenborg, H., Münstermann, G., Knüpfer, M. M., Hotfilder, M., and Wolff, J. E. (1997). C6 cells cross-resistant to cisplatin and radiation. Anticancer Res. 17, 2073-2077.

Ryan, R. M., Compton, E. L. R., and Mindell, J. A. (2009). Functional characterization of a Na+-dependent aspartate transporter from Pyrococcus horikoshii. J. Biol. Chem. 284, 17540-17548. doi:10.1074/ jbc.M109.005926

Sonawane, V. R., Siddique, M. U. M., Gatchie, L., Williams, I. S., Bharate, S. B., Jayaprakash, V., et al. (2019). CYP enzymes, expressed within live human suspension cells, are superior to widely-used microsomal enzymes in identifying potent CYP1A1/CYP1B1 inhibitors: identification of quinazolinones as CYP1A1/CYP1B1 inhibitors that efficiently reverse $\mathrm{B}[\mathrm{a}] \mathrm{P}$ toxicity and cisplatin resistance. Eur. J. Pharm. Sci. 131, 177-194. doi:10.1016/j. ejps.2019.02.016

Sun, J., Nagel, R., Zaal, E. A., Ugalde, A. P., Han, R., Proost, N., et al. (2019). SLC 1A3 contributes to L-asparaginase resistance in solid tumors. Embo J. 38, e102147. doi:10.15252/embj.2019102147

Tao, J., Deng, N. T., Ramnarayanan, K., Huang, B., Oh, H. K., Leong, S. H., et al. (2011). CD44-SLC1A2 gene fusions in gastric cancer. Sci. Translational Med. 3, 77ra30. doi:10.1126/scitranslmed.3001423

Vandenberg, R. J., and Ryan, R. M. (2013). Mechanisms of glutamate transport. Physiol. Rev. 93, 1621-1657. doi:10.1152/physrev.00007.2013

Varamo, C., Peraldo-Neia, C., Ostano, P., Basiricò, M., Raggi, C., Bernabei, P., et al. (2019). Establishment and characterization of a new intrahepatic cholangiocarcinoma cell line resistant to gemcitabine. Cancers 11, 519. doi:10.3390/cancers11040519

Wang, D., and Lippard, S. J. (2005). Cellular processing of platinum anticancer drugs. Nat. Rev. Drug Discov. 4, 307-320. doi:10.1038/nrd1691

Xu, L., Chen, J., Jia, L., Chen, X., Awaleh Moumin, F., and Cai, J. (2020). SLC1A3 promotes gastric cancer progression via the PI3K/AKT signalling pathway. J. Cel. Mol. Med. 24, 14392-14404. doi:10.1111/jcmm.16060

Ye, Z.-C., Rothstein, J. D., and Sontheimer, H. (1999). Compromised glutamate transport in human glioma cells: reduction-mislocalization of sodiumdependent glutamate transporters and enhanced activity of cystineglutamate exchange. J. Neurosci. 19, 10767-10777. doi:10.1523/JNEUROSCI 19-24-10767.1999 
Zhang, Y., Wang, Z., Yu, J., Shi, J. z., Wang, C., Fu, W. h., et al. (2012). Cancer stemlike cells contribute to cisplatin resistance and progression in bladder cancer. Cancer Lett. 322, 70-77. doi:10.1016/j.canlet.2012.02.010

Zhu, R., Xue, X., Shen, M., Tsai, Y., Keng, P. C., Chen, Y., et al. (2019). NFkB and $\mathrm{TNF} \alpha$ as individual key molecules associated with the cisplatin-resistance and radioresistance of lung cancer. Exp. Cel Res. 374, 181-188. doi:10.1016/j.yexcr. 2018.11.022

Zhuang, H., Shi, S., Guo, Y., and Wang, Z. (2019). Increase of secondary mutations may be a drug-resistance mechanism for lung adenocarcinoma after radiation therapy combined with tyrosine kinase inhibitor. J. Cancer. 10, 5371-5376. doi:10.7150/jca.35247
Conflict of Interest: The authors declare that the research was conducted in the absence of any commercial or financial relationships that could be construed as a potential conflict of interest.

Copyright $\odot 2021 \mathrm{Hao}, \mathrm{Wu}, \mathrm{Cao}, \mathrm{Yu}$, Ning, Wang, Lin and Chen. This is an openaccess article distributed under the terms of the Creative Commons Attribution License (CC BY). The use, distribution or reproduction in other forums is permitted, provided the original author(s) and the copyright owner(s) are credited and that the original publication in this journal is cited, in accordance with accepted academic practice. No use, distribution or reproduction is permitted which does not comply with these terms. 